In our editorial, we focused on discussing $D r$. Duncan's paper and fast-tracking techniques. We did not compare his work to other fast-tracking studies. References (the number of which is limited to ten) were selected to underline specific points in the discussion. Notwithstanding Dr. White's considerable contributions to the field of fast-tracking in ambulatory anesthesia, because of the limited space and a different focus, these could not be included in our editorial comment on Dr. Duncan's study. Dr White can rest assured that it was never our intent to "knowingly ignore the peer-reviewed literature on a topic when preparing an editorial".

Dajun Song MD PhD

Frances Chung FRCPC

Toronto, Ontario

\section{Epidural analgesia and maternal fever}

To the Editor:

We read with interest the article by Vallejo et al. ${ }^{1}$ and wish to comment on the methodology used and the authors' conclusions.

In this case-control study patients were selected from database in which the presence of maternal fever $>38.0^{\circ} \mathrm{C}$ was a diagnostic sign for chorioamnionitis. Thus, it is hardly surprising that $100 \%$ of the selected patients had fever (regardless of whether they had an epidural or not), while only $1 \%$ of the patients enrolled in the no-chorioamnionitis group developed fever. Accordingly, the chorioamnionitis patients had also a higher incidence of histologic chorioamnionitis. In addition, since the indication for neonatal sepsis evaluation rate was maternal fever or clinical amnionitis, the differences in evaluation rates precisely followed patients' selection rather than - as implied from the discussion section - a new finding.

Based on the above, we question the authors' conclusion that chorioamnionitis and not epidural anesthesia was the cause for maternal fever, a finding that could not have been derived from the methodology that was used.

The incidence of maternal fever increases with longer epidural use, ${ }^{2,3}$ ranging between $7 \%$ with epidural use less than six hours to $36 \%$ after $>18 \mathrm{hr}$. The authors did not report labour length, thus, the low incidence of maternal fever in the epidural without amnionitis group could relate to a short epidural use.

We believe that a large-scale prospective study that examines maternal and neonatal outcome, together with histological and microbiological evaluations of the placenta and neonate, would better elucidate the true nature of maternal fever after epidural analgesia for labour.

Yitzhak Cohen MD

Tel Aviv, Israel

\section{References}

1 Vallejo MC, Kaul B, Adler LJ, et al. Chorioamnionitis, not epidural analgesia, is associated with maternal fever during labour. Can J Anesth 2001; 48: 1122-6.

2 Lieberman E, Lang JM, Frigoletto F Jr, Richardson DK, Ringer $S A$, Cohen A. Epidural analgesia, intrapartum fever, and neonatal sepsis evaluation. Pediatrics 1997; 99: 415-9.

3 Fusi L, Maresh MJA, Steer PJ, Beard RW. Maternal pyrexia associated with the use of epidural analgesia in labour. Lancet 1989; 1: 1250-2.

\section{REPLY:}

The selected variable in our obstetrical database was clinical chorioamnionitis (amnionits), and not fever (> $38^{\circ} \mathrm{C}$ ). Our results illustrate the relationship between fever and amnionitis in that parturients with the diagnosis of amnionits with or without concomitant labour epidural analgesia (LEA; Groups I and II) have a significantly bigher percentage of fever (100\%) compared to the LEA group without concomitant amnionitis (1\% Group III). ${ }^{l}$ Indeed, these results were due to patient selection and the diagnosis of histological chorioamnionitis is biased due to the selection process. However, the author of the letter has overlooked the main point of patient selection (methodology). Parturients were selected to control for the confounding effect clinical chorioamnionitis (amnionitis) has on maternal fever and LEA. Our methodology purposefully subdivided nulliparous parturients into three groups to control for the presence of clinical chorioamnionitis (amnionitis). Clearly, when parturients are subdivided by whether they presented with amnionitis or not, the numbers of non-amnionitis parturients with maternal fever $\left(>38^{\circ} \mathrm{C}\right)$ drops to almost zero $(P=0.000){ }^{1}$

The incidence of maternal fever increases with duration of epidural use, about $0.07^{\circ} \mathrm{C}$ per hour. ${ }^{2}$ However, epidural analgesia does not elevate maternal temperature enough to cause maternal fever $\left(>38^{\circ} \mathrm{C}\right)$ regardless of labour duration. ${ }^{2,3}$ Additionally, our results concur with the results of Dashe et al. in that epidural analgesia is associated with intrapartum fever only in the presence of histologic chorioamnionitis. ${ }^{4}$

We too believe a large-scale prospective study is necessary to better elucidate the true nature of maternal fever, however, the present study suggests that one must control for and cannot ignore the confounding factor of clinical chorioamnionitis (amnionitis). 